\title{
THE FINITARY ISOMORPHISM THEOREM FOR MARKOV SHIFTS
}

\author{
BY M. KEANE AND M. SMORODINSKY
}

Introduction. The results announced here are concerned with the isomorphism theory of dynamical systems. Many dynamical systems occur in nature, and although they are by definition deterministic processes, they often exhibit (what seems to be) random behavior to the observer. One of the major motivations of ergodic theory is to display isomorphisms between deterministic processes and processes which are clearly random in nature, such as Bernoulli shifts or Markov processes. To have physical significance, these isomorphisms should be continuous in some sense, and the suitable concept turns out to be that of finitary isomorphism, defined below. This research provides a basic item for the development of the above ideas, in showing that two irreducible Markov processes with the same information content per unit time (= entropy) are finitarily isomorphic. That is, there is really only one type of stationary random behavior.

The adjective finitary means that the map of one sequence space to the other is almost surely continuous in the sense that a finite section of the image sequence is determined by a sufficiently long finite section of the original sequence; generally, the length required depends upon the particular sequence on hand.

THEOREM 1. Irreducible finite memory Markov shifts on a finite or countable state space and of the same entropy and period are finitarily isomorphic.

It is easy to see that without loss of generality we can assume that the Markov shifts are of memory one. Also, since a Markov shift of period $d$ is a direct product of $d$ points with a mixing Markov shift, the base being a finite or countable union of cylinder sets, we can assume mixing. The proof of Theorem 1 makes use of the following.

LEMmA. Let $\left\{X_{n}\right\}$ be a stationary mixing Markov process of a finite or countable state space $A=\left\{a_{1} a_{2} \cdots\right\}$. Then, there are two stationary processes $\left\{Y_{n}\right\},\left\{Z_{n}\right\}$ on finite or countable state spaces $B=\left\{b_{1} b_{2} b_{3} \cdots\right\}$ and $C=$ $\left\{c_{1} c_{2} c_{3} \cdots\right\}$ with at least three states each, and of the same entropy as $\left\{X_{n}\right\}$,

Received by the editors July 14, 1978.

AMS (MOS) subject classifications (1970). Primary 28A65.

(C) 1979 American Mathematical Society 0002-9904/79/0000-0113/\$01.75 
$\left\{Y_{n}\right\}$ being an independent process, and the following hold:

(i) There is a natural $k$ such that the two processes

$$
W_{n}=\left\{\begin{array}{l}
d_{1} \text { if } \exists i, Y_{i}=Y_{i=1}=\cdots=Y_{i+k-1}=b_{1} \text { and } i \leqslant n \leqslant i+k-1 \\
d_{2} \text { otherwise, }
\end{array}\right.
$$

and

$$
\widetilde{W}_{n}=\left\{\begin{array}{l}
d_{1} \text { if } Z_{n}=c_{3} \\
d_{2} \text { otherwise }
\end{array}\right.
$$

have the same distribution.

(ii) The two processes

$$
V_{n}=\left\{\begin{array}{l}
e_{1} \text { if } X_{n}=a_{1} \\
e_{2} \text { otherwise }
\end{array}\right.
$$

and

$$
\widetilde{V}_{n}=\left\{\begin{array}{l}
e_{1} \text { if } Z_{n}=c_{1} \\
e_{2} \text { otherwise }
\end{array}\right.
$$

have the same distribution.

(iii) $c_{1}$ and $c_{3}$ are each renewal states for the process $Z_{n}$.

The proof of Theorem 1 now proceeds along the following lines. We use our Marker Method of [7] and [8] to construct a finitary isomorphism between $\left\{X_{n}\right\}$ and $\left\{Z_{n}\right\}$ and also between $\left\{Z_{n}\right\}$ and $\left\{Y_{n}\right\}$. Since $\left\{Y_{n}\right\}$ is a Bernoulli scheme and Bernoulli schemes are finitarily isomorphic, the proof is complete.

An interesting corollary of Theorem 1 is the following.

THEOREM 2. Given an ergodic automorphism of the two-dimensional torus, there is a partition into sets with nonempty interior and zero measure boundary which is independent under the action of the automorphism.

It is interesting to compare this with Bowen's result [5]. Adler and Marcus [3] recently proved that topological Markov shifts with the measure of maximal entropy are finitarily isomorphic.

Another result that follows from Theorem 1 is

THEOREM 3. $\beta$-automorphisms are finitarily isomorphic to Bernoulli shifts.

See [9] for comparison.

Details of proofs will appear elsewhere. 


\section{REFERENCES}

1. M. A. Akcoglu, A. del Junco and M. Rahe, Finitary codes between Markov processes (to appear).

2. R. L. Adler, P. Shields and M. Smorodinsky, Irreducible Markov shifts, Ann. Math. Statist. 43 (1972), 1027-1029.

3. R. L. Adler and B. Marcus, Topological entropy and equivalence of dynamical systems (to appear).

4. R. Adler and B. Weiss, Similarity of automorphisms of the torus, Mem. Amer. Math. Soc. No. 98 (1970).

5. R. Bowen, Smooth partitions of Anosov diffeomorphism are weak Bernoulli, Israel J. Math. 21 (1975), 95-100.

6. N. Friedman and D. S. Ornstein, On isomorphism of weak Bernoulli transformations, Advances in Math. 5 (1971), 354-394. $352-371$.

7. M. Keane and M. Smorodinsky, A class of finitary codes, Israel J. Math. 26 (1977), of Math. (to appear).

9. M. Smorodinsky, $\beta$-Automorphisms are Bernoulli shifts, Acta Math. Acad. Sci. Hungar. 24 (1973), 273-278.

\section{ISRAEL}

DEPARTMENT OF STATISTICS, UNIVERSITY OF TEL AVIV, TEL AVIV,

U.E.R. MATHEMATIQUES ET INFORMATIQUE, UNIVERSITE DE RENNES I, AVENUE DU GENERAL LECLERC, 35042 RENNES CEDEX, FRANCE 